\title{
Pulse characteristics of passively mode-locked diode lasers
}

\author{
Randal A. Salvatore, Thomas Schrans, and Amnon Yariv \\ Department of Applied Physics 128-95, California Institute of Technology, Pasadena, California 91125
}

Received December 9, 1994

\begin{abstract}
For the first time to our knowledge, asymmetric pulse shapes and the linear and nonlinear chirp from a passively mode-locked semiconductor laser are directly measured. For the laser tuned to various center wavelengths, falltime-to-rise-time ratios of 2.0 to 2.5 are measured. With the laser tuned to the shorter-wavelength side of its tuning range, a significant quadratic chirp of $-60 \mathrm{fs} / \mathrm{nm}^{2}$ is measured, along with a linear chirp of $-800 \mathrm{fs} / \mathrm{nm}$. The nonlinear chirp is responsible for the asymmetrically shaped compressed pulses that produce long-tailed autocorrelations.
\end{abstract}

In the generation of ultrashort laser pulses, knowledge of the complex pulse envelope, i.e., both amplitude and phase behavior, of the pulses leaving the laser is a key to achieving optimally short pulses. As early as 1980 passively mode-locked semiconductor lasers were known to generate picosecond, although not time-bandwidth-limited, optical pulses. In 1985 it was concluded that the excess time-bandwidth product of these typical picosecond pulses was due to an upchirp (an increasing optical frequency during each pulse). ${ }^{1} \quad$ Often the chirp is extremely linear and thus easily compensated by a dual-grating pulse compressor, permitting generation of $<300$-fs pulses. ${ }^{2-4}$ Under other conditions, when tuned to different wavelengths or biased differently, the laser does not produce such a linear chirp. We describe general trends of the passively mode-locked laser's pulse shape and chirp as the laser's spectrum is tuned to different wavelengths in the tuning range.

Standard autocorrelation measurements preclude extraction of information concerning the pulse shape and chirp. Most techniques for measuring pulse shape or chirp require algorithmic methods involving interferometric field autocorrelations $s^{5}$ or are useful only for higher-energy pulses ( $1 \mathrm{~nJ}$ or more). ${ }^{6}$ In this Letter we use a direct measurement of pulse shape and a direct measurement of the chirp similar to that used by Chilla and Martinez for dye lasers. ${ }^{7}$ It is demonstrated that, for passively mode-locked semiconductor lasers, the asymmetric pulse shape, linear chirp, and second-order chirp can be measured by use of cross-correlation and filtering techniques.

To measure the pulse shape, we used the setup shown in Fig. 1. The laser is a quadruple-quantumwell two-section GaAs buried heterostructure, as described previously. ${ }^{8}$ Because the pulses coming directly from the laser are naturally stretched out and can easily be compressed by at least an order of magnitude, we split off part of the beam and send it into a dual-grating compressor (without spatial filter), thus making it effectively a delta function. Next, the two beams are collinearly recombined and sent into a second-harmonic-generation crystal, and their temporal cross correlation is measured. The resulting cross correlation will thus be the shape of the intensity envelope of the original pulse leaving the laser.
The results of the measurement are shown in Fig. 2. The asymmetry of the pulses is readily visible. To quantify this, we measured the 10-90\% rise and fall times of the pulse intensity. When the laser is tuned near the center or longer wavelengths of the tuning range $(838-854 \mathrm{~nm}){ }^{3}$ the ratio of the fall time to the rise time is approximately $2.3: 1$ [Fig. 2(a)]. The pulse shape for the laser tuned to the shorter-wavelength side of the tuning range is shown (for a 841-nm center wavelength) in Fig. 2(b). In this case a more sharply peaked pulse is measured but with tails extending slightly further. Similarly, the ratio of the fall time to the rise time was found to be $2.0: 1$.

The asymmetry in the resulting pulse shapes is explained by the fact that, in the passively modelocked laser, both the rising and falling edges are shaped by different physical phenomena. The rising or front edge of the pulse is shaped by the loss of the absorber section until it is saturated, whereas the falling edge of the pulse is shaped as the gain section undergoes enough saturation to again present a net loss. ${ }^{9} \quad$ The absorber has a relatively large (in magnitude) differential absorption $A=-\mathrm{d} \alpha / \mathrm{d} N_{a}$, which means that each photon absorbed reduces the absorption rather effectively. This helps to provide a relatively fast saturation process. The saturation rate, from the carrier rate equation, is given by

$$
\frac{\mathrm{d} N_{a}(t)}{\mathrm{d} t}=-\Gamma A N_{a}(t) P(t),
$$

where $N_{a}(t)$ is the density of carriers contributing to absorption, $\Gamma$ is the confinement factor, and $P(t)$ is the photon flux.

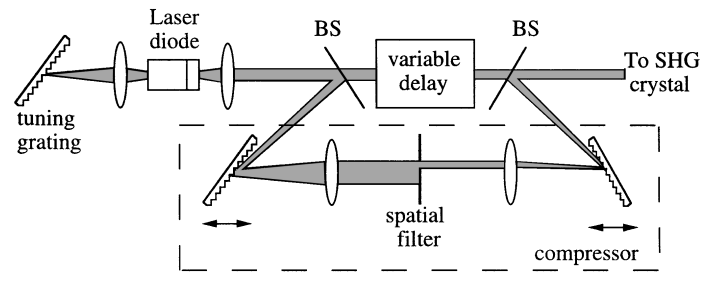

Fig. 1. Setup of the cross-correlation system for measurement of the intensity envelope of pulses. An adjustable-position spatial filter is used in the Fourier plane for spectral filtering in chirp measurements only. BS's, beam splitters; SHG, second-harmonic-generation. 

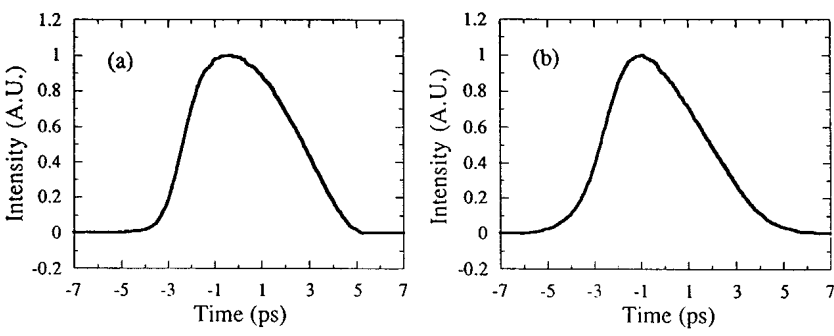

Fig. 2. Measured shape of the pulse leaving the semiconductor laser for the spectrum center tuned to (a) $848 \mathrm{~nm}$ and (b) $841 \mathrm{~nm}$.

On the other hand, since differential gain typically saturates with higher population, the differential gain $G=\mathrm{d} g / \mathrm{d} N_{g}$ is smaller by approximately a factor of 2 , resulting in a larger saturation energy and a slower saturation rate:

$$
\frac{\mathrm{d} N_{g}(t)}{\mathrm{d} t}=-\Gamma G N_{g}(t) P(t),
$$

where $N_{g}(t)$ is the density of carriers contributing to gain. The gain section's slower saturation rate can account for the slower shaping at the falloff of the optical pulses. To double check the pulse shape results, we have numerically generated an autocorrelation of a pulse from Fig. 2 and compared the result with the actual measured autocorrelation taken under the same conditions. The two resulting plots match well, indicating that the compressed pulse is close enough to a delta function and does not introduce significant broadening.

The setup for measuring the spectral phase is identical to the setup for measuring the pulse shape (Fig. 1), except now a single-slit spatial filter is added in the Fourier plane between the two lenses of the dual-grating compressor to pass a narrow slice of the spectrum. ${ }^{10}$ The full field spectrum may be described as $F(\omega) \exp \left[-j \tau_{n}\left(\omega-\omega_{0}\right)\right]$, where $F(\omega)$ represents the magnitude of spectrum, $w_{0}$ is the spectrum's center frequency, and $\tau_{n}$ is the delay for slice $n$ and can vary across the spectrum, thus accounting for any spectral phase. On filtering, a slice of the spectrum having center frequency $\omega_{f}$ and filter width $\Delta \omega_{f}$ (assuming a Gaussian filter for simplicity) is then

$$
F(\omega) \exp \left[-j \tau_{n}\left(\omega-\omega_{0}\right)\right] \exp \left[\frac{-\left(\omega-\omega_{f}\right)^{2}}{\left(\Delta \omega_{f}\right)^{2}}\right] .
$$

Taking the transform of this expression (to within a constant), one can see that $\tau_{n}$ exactly represents the group delay of the pulse from any narrow slice of the spectrum:

$$
\left\{f\left(t-\tau_{n}\right) * \exp \left[\frac{-\left(\Delta \omega_{f}\right)^{2}\left(t-\tau_{n}\right)^{2}}{4}\right]\right\} \exp \left(j \omega_{f} t\right) .
$$

If $\tau_{n}$ is a nonzero constant throughout the spectrum, this adds a uniform time delay to the pulse, as seen from the above convolution. If $\tau_{n}$ varies linearly or quadratically with $n$, a broadening or asymmetry, respectively, of the pulse results.
Using this setup and choosing any slice of the spectrum, we can measure the corresponding time delay for the slice's center wavelength. By measurement of the peak's position in the cross correlation the group delay $\tau_{n}$ can be determined for different wavelengths, and thus the sign and the magnitude of the chirp can be measured. For even more accuracy in measuring the nonlinear chirp we send both beams through the compressor (using cylindrical lenses to keep the beams distinct), delaying one and filtering the other, and using the proper grating spacing to remove the linear part of $\tau$ versus $\lambda$.

The directly measured curves for the crosscorrelation delay versus slice center wavelength are plotted in Fig. 3 based on 1.2-nm slices. The chirp in Fig. 3(a) is extremely linear, meaning that it would be ideal for compensation from a dual-grating pulse compressor. Results from pulse compression confirm this, since this is the case in which a minimum compressed near-transform-limited pulse width of $260 \mathrm{fs}$ is achieved. ${ }^{3}$ Even when we phase compensate both beams by sending each through the compressor, eliminating the linear chirp, no nonlinear chirp can be detected; only a linear chirp of $-900 \mathrm{fs} / \mathrm{nm}$ is measured. Resolution for the measurement of nonlinear chirp is estimated to be $\pm 10 \mathrm{fs} / \mathrm{nm}^{2}$. The dual-grating compressor is expected to add a negligible nonlinear chirp of $5 \mathrm{fs} / \mathrm{nm}^{2}$.

The chirp resulting from the laser tuned to the shorter-wavelength side of the tuning range $(841 \mathrm{~nm})$ has a significant nonlinear component. The $\tau$ versus $\lambda$ curve shows a steeper slope at the longwavelength side of the spectrum, and the linear chirp was measured to be slightly smaller in magnitude $(-800 \mathrm{fs} / \mathrm{nm})$. More accurately measuring the nonlinear chirp by removing most of this linear chirp, as described previously, we obtain a nonlinear chirp of $-60 \pm 10 \mathrm{fs} / \mathrm{nm}^{2}$. Higher-order terms in the nonlinear chirp are not found to be present. The external cavity grating that is rotated for tuning is known to add no dispersion by itself and may add dispersion only as a result of lens aberrations that are considered negligible here; therefore the nonlinear chirp results from the semiconductor laser itself. The same long-tailed autocorrelations will result from the laser when the feedback from an external cavity mirror (used in place of the grating) is reduced. Thus the long tails and nonlinear chirp do
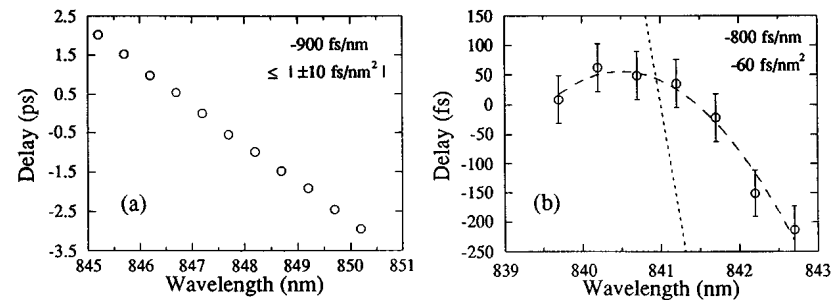

Fig. 3. Measurement of the chirp in terms of group delay versus the rectangular filter's center wavelength (dashed curve), yielding (a) $-900 \mathrm{fs} / \mathrm{nm}$ and $\pm 10 \mathrm{fs} / \mathrm{nm}^{2}$ for the laser tuned to $848 \mathrm{~nm}$ and (b) a nonlinear chirp (dashed curve) of $-60 \mathrm{fs} / \mathrm{nm}^{2}$ for the laser tuned to $841 \mathrm{~nm}$. The linear chirp of $-800 \mathrm{fs} / \mathrm{nm}$ is shown for comparison (dotted curve). 

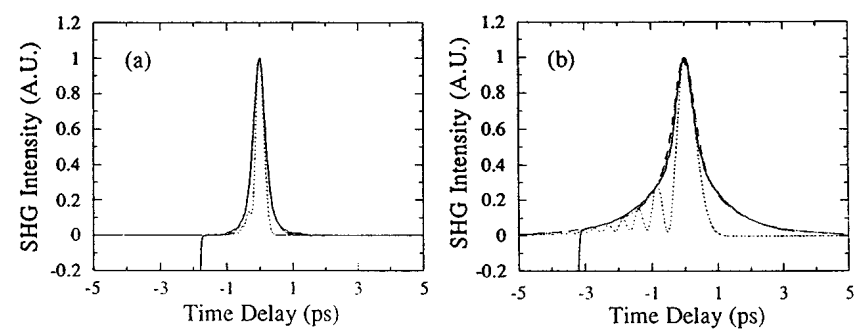

Fig. 4. Fit of the calculated (dashed curves) to the measured (solid curves) autocorrelation of the compressed pulse and the calculated compressed pulse intensity resulting from the fit (dotted curves) for the laser tuned to (a) $848 \mathrm{~nm}$ and (b) $841 \mathrm{~nm}$. The fit shows that the nonlinear chirp is mainly quadratic.

not seem to be an inherent result of the tuning but a result of larger self-phase-modulation nonlinearities induced through the deeper cycling of the gain section. In these measurements, at $\sim 1-\mathrm{mW}$ average optical power and $600-\mathrm{MHz}$ pulse repetition, smaller time-bandwidth products and slightly less linear chirp (in terms of $\mathrm{d} \tau / \mathrm{d} \lambda$ ) are obtained from lower- $Q$ cavities, even though higher peak powers and more nonlinear chirp are present.

As is known from studies of semiconductor laser amplifiers, the linewidth enhancement factor $\alpha(w, N)$ that couples amplitude to phase will cause a frequency chirp across the pulse. ${ }^{11}$ The effect of the changing index over some length of gain material is not unlike the effect that one would expect from a Doppler red shift as the optical path length is increased. Assuming a relationship between the gain and the change in index, $n(t)=\alpha[N(t), w] g(t)$, the phase shift on traveling through a length $L_{g}$ of laser material is

$$
\phi(t)=\omega t-k_{0} L_{g}\left[n_{0}+\frac{\mathrm{d} n(t)}{\mathrm{d} t} t\right],
$$

and by use of Eq. (2) the self-phase-modulation frequency shift is found to be approximately

$$
\Delta \omega(t)=-k_{0} \alpha\left[\omega, N_{g}(t)\right] \Gamma^{2} G^{2} N_{g}(t) L_{g} P(t) .
$$

In our measurements the self-phase modulation, in terms of the magnitude of $\mathrm{d} \tau / \mathrm{d} \lambda$, from the gain section significantly dominates the equivalent term that results from the absorber section. The half-way saturation of $N_{g}(t)$ and, even more so, the peak frequency shift is expected to occur before the pulse reaches its peak intensity. The upward concavity in a plot of $\omega(t)$ near the pulse's peak is consistent with the measured sign of nonlinear chirp. Although a self-consistent solution with these large nonlinearities must be found to explain conclusively the extremely linear chirp, it is likely that, during the peak of the pulse, a rather linear upchirp occurs.

To verify and extract further information, we have simulated numerically the pulse-and-compression system. Using the pulse shape measurements and estimated linear and quadratic chirp and requiring that the resulting spectrum match the measured spectrum well, we calculated the resulting uncom- pressed and compressed pulse autocorrelations and found them to be in good agreement with the measurements. In Fig. 4(a) the solid curve is the measured autocorrelation for the compressed pulse for the laser tuned to $848 \mathrm{~nm}$, and a simulation with $-800 \mathrm{fs} / \mathrm{nm}$ and $-15 \mathrm{fs} / \mathrm{nm}^{2}$ gives an autocorrelation that matches well (indiscernible in the plot). According to the simulation, the dotted curve represents the actual pulse intensity envelope that we would expect to measure for the compressed pulse. Figure 4(b) shows the corresponding simulation for the laser operating at $841 \mathrm{~nm}$. Again, the solid curve represents the measured autocorrelation for the compressed pulse. The dashed curve is the simulation and matches the long tails well, with $-700 \mathrm{fs} / \mathrm{nm}$ and $-70 \mathrm{fs} / \mathrm{nm}^{2}$ chosen for the pulse in this case. The expected envelope of the compressed pulse is displayed by the dotted curve. The nonlinear chirp can adequately explain the long tails remaining after pulse compression. From the measured sign of the nonlinear chirp it is suspected that small lobes precede the actual compressed subpicosecond pulses in time.

In conclusion, the shape of pulses leaving the modelocked semiconductor laser has been directly measured, and its asymmetry is attributed to the slower saturation rate of the gain section compared with the absorber section. Linear and nonlinear chirp have been measured for various center wavelengths. The nonlinear chirp is associated with reduced external cavity feedback, which has been known to force deeper cycling of the gain section and shift the linear segment of the self-phase modulation away from the pulse center, and is seen to leave large tails, limiting the minimum pulse width of the dual-gratingcompressed pulse.

This study was supported by the National Science Foundation under grant ECS-9001272 and by the U.S. Office of Naval Research under grant N0001491-J-1195.

\section{References}

1. Y. Silberberg and P. W. Smith, IEEE J. Quantum Electron. QE-22, 759 (1986).

2. P. J. Delfyett, L. Florez, N. Stoffel, T. Gmitter, N. Andreadakis, G. Alphonse, and W. Ceislik, Opt. Lett. 17, 670 (1992).

3. R. A. Salvatore, T. Schrans, and A. Yariv, IEEE Photon. Technol. Lett. 5, 756 (1993).

4. N. Stelmakh and J-M. Lourtioz, Electron. Lett. 29, 161 (1993).

5. P. Simon, N. Gerhardt, and S. Szatmari, Opt. Quantum Electron. 23, 73 (1991).

6. D. J. Kane and R. Trebino, IEEE J. Quantum Electron. 29, 571 (1993).

7. J. L. A. Chilla and O. E. Martinez, IEEE J. Quantum Electron. 27, 1228 (1991).

8. S. Sanders, A. Yariv, J. Paslaski, J. E. Ungar, and H. A. Zarem, Appl. Phys. Lett. 58, 681 (1991).

9. H. Haus, IEEE J. Quantum Electron. QE-11, 736 (1975).

10. A. M. Weiner, J. P. Heritage, and E. M. Kirschner, J. Opt. Soc. Am. B 5, 1563 (1988).

11. A. Dienes, J. P. Heritage, M. Y. Hong, and Y. H. Chang, Opt. Lett. 17, 1602 (1992). 